\title{
Intraarticular Osteoid Osteoma of the Hip with Lengthening of Limb Secondary to Proximal Femoral Hypertrophy: A Case Report
}

\author{
Anoop $\mathrm{S}^{1}$; Saem $\mathrm{S}^{2}$; Karen D'sa ${ }^{3}$; Shenoy R M ${ }^{4}$. \\ ${ }^{1 \& 2-}$ Junior Residents, ${ }^{3}$ senior Resident, ${ }^{4}$ profesor \& Unit Head Orthopaedic Department, Yenepoya Medical \\ College Derlakattae,Mangalore Karnataka, India.
}

\begin{abstract}
: 12-year-old boy presented with pain in the right hip since $11 / 2$ years, with associated apparent lengthening of the limb and limp since 9 months. He was thoroughly examined and investigated clinically and radiologically. A diagnosis of a long standing osteoid osteoma $(\mathrm{OO})$ in the neck of femur resulting in hypertrophy of the proximal femur and $1 / 2$ an inch true lengthening of the limb was made. He underwent an En bloc excision of the tumour. The pain relief was noticed immediately after surgery and there was no sign of recurrence at one year follow-up. This case of intra articular $O O$ is presented herewith because of a long standing and interesting presentation causing hypertrophic structural changes in the proximal femur, resulting in true limb lengthening and a limp which to our knowledge has not been reported so far.
\end{abstract}

Keywords: Osteoid osteoma, Intracapsular, Pathological limb lengthening, Benign tumor Hip.

\section{Introduction}

Osteoid osteoma (OO) is a relatively common skeletal lesion that accounts for approximately $12 \%$ of benign skeletal neoplasms. Bergstrand in 1930 was the first who described the pathological findings ${ }^{1}$. However, Jaffe in 1935 was the first to recognize osteoid osteoma as a separate entity, in a report of five cases. Since then, more than 1,000 cases have been reported in the literature, establishing it as a common benign lesion ${ }^{1}$.

For physicians, most of $\mathrm{OO}$ no longer presents a diagnostic problem, because the clinical, radiologic and scintigraphic characteristics have been well described. The classic symptoms, however, may not always be present, or they can be misleading and altered, especially when the tumor is located intra articularly. Because of the unusual clinical and radiological features of this entity, intraarticular osteoid osteoma (IAOO) poses a diagnostic challenge ${ }^{2}$.

There is general agreement in the literature that complete excision is the treatment of choice and that incomplete removal of the nidus leads to recurrence of symptoms ${ }^{3}$. Recently, minimally invasive techniques such as computed tomography (CT) - guided core-drill excision, arthroscopic removal, cryo ablation and thermo ablation by laser or radiofrequency energy have emerged as alternatives to the conventional surgical excision. However, the success rates of these methods ranges from $70-100 \%{ }^{4}$.

\section{Case presentation}

A 12 year old male presented with pain in the right hip of $1 \frac{1}{2}$ years with gradually developing limp of 9 months. Pain was continuous, worse at night, relieved by non steroidal anti inflammatory drugs (NSAID), which the patient has been taking for $1 \frac{1}{2}$ years, intermittently. Physical examination revealed painless movements of the hip with limitation in terminal degrees. Type of gait was painless limping and neurologic examination did not reveal any abnormality.

Blood count and biochemical profile were within the reference range.

The radiograph showed thickened bone in the anteromedial aspect of neck of right femur with sclerosis and periosteal reaction along with an oval nidus surrounded by a radiolucent rim. The medial joint space was increased and the lateral part of femoral head was uncovered, with increase in the size of capital femoral epiphysis. The proximal femur showed hypertrophic changes (Figure 1,2,3). Computed tomography and magnetic resonance imaging of the right hip joint revealed a lesion at the anteromedial portion of the femoral neck with nidus of about $0.7 \mathrm{~cm}$ suggestive of osteoid osteoma. (Figure 4,5).

An En bloc surgical excision of the lesion was done under general anaesthesia and under $\mathrm{C}$ arm guidance. An anterolateral approach to the hip joint was used and the femoral neck region was exposed dissecting between tensor fascia lata medially and gluteus medius laterally. On opening the capsule, synovial fluid with flakes of inflammed tissue leaked out (Figure 6). After clearing the same from the joint, localization of the lesion, intra-operatively, was achieved by using Kirschner wire as marker under ' $\mathrm{C}$ ' arm guidance. The block for excision was marked by drilling holes along the margins. The bone was oteotomised using a small osteotome along the drill holes and the lesion was excised en block and the same was confirmed under c-arm 
(Figure 7,8,9).The exposure was closed in layers over a suction drain. Histological examination of the excised tissue, confirmed the diagnosis of OO (Figure 10). Normal bone histology in the area adjacent to OO confirmed complete excision of the lesion.

Post-operatively, the limb was protected for 3 weeks with non weight bearing and then gradual weight bearing as tolerated was allowed for next three weeks. Patient had significant pain relief during immediate postoperative period. At the end of 6 weeks, he returned to his previous level of physical activity. Follow up at the end of 1 year did not show presence of any residual symptoms or recurrence, clinically and radiologicaly (Figure 11). Limb lengthening however persisted with an insignificant limp.

\section{Discussion}

$\mathrm{OO}$ is a benign bone-forming tumor that involves cortical or cancellous bone. The lesion is usually less than $1.5 \mathrm{~cm}$ in size ${ }^{2}$. In 1966, Edeiken et al categorized three types of osteoid osteoma, each with varying amounts of associated osteosclerosis depending on the variety. Cortical shows maximum sclerosis when compared to cancellous and sub periosteal variety. The lesion is most commonly located in the cortical bones and less often in the cancellous. Cancellous lesions are frequently intraarticular and may be associated with synovitis and joint effusion. Rarely, OO occurs in a subperiosteal location ${ }^{5}$. Patients are usually young with strong male predominance. As broadly defined by Kattapuram et al, OO occurring at the ends of long bone, in and around the joint either in that part of the bone surrounded by capsule and synovium or very close to the capsule and synovium will be considered IAOO ${ }^{6}$.

The lesion may occur in any bone. However, there is a predilection for the lower extremity with $50 \%$ or more of lesions occurring in the femur and tibia ${ }^{7}$. Pain is the leading symptom of the lesion. It is usually described as mild and intermittent at first, later becoming more constant and severe. Before the lesion is apparent radiologically, some cases may present with intense pain. Pain is frequently worse at night and may awaken the patient from sleep and often is relieved by aspirin or other NSAID ${ }^{4}$. IAOO in and around the hip can mimic a hip pathology as it produces synovitis and effusion. When it is located in a portion of the bone that is within the capsule of a joint, an OO may present with clinical findings that are more indicative of an inflammatory synovitis. Chronic changes of bone remodelling, such as widening and foreshortening of the femoral neck with changes in the height of capital femoral epiphysis can be seen if the diagnosis is delayed for more than 3 months ${ }^{8}$.

IAOO can be difficult to locate radiologically, when in doubt bone scintigraphy followed by CT is the investigation of choice.

The imaging modality of choice for identifying the nidus of an OO is CT. A CT scan usually gives a sharp contrast between the nidus and the surrounding normal trabecular bone even in the absence of a sclerotic rim. Rarely, CT results may be falsely negative. On the other hand, MRI is less useful than CT, and may miss small lesions, or show nonspecific changes ${ }^{9}$. Further, soft tissue and bone edema around the nidus can be confusing and can suggest an aggressive or malignant process.

Initial treatment of $\mathrm{OO}$ involves the use of NSAIDs or aspirin ${ }^{10}$. Complete surgical en bloc excision of the $\mathrm{OO}$ nidus is curative and brings immediate and dramatic relief of symptoms, and is still the preferred treatment method ${ }^{11}$. The clinical success rate of surgery ranges from 88 to $100 \%{ }^{12}$. Nevertheless, difficulties faced at surgical excision in accessing and localizing the lesion has made the recent minimally invasive interventions more appealing as methods of treatment. Less invasive methods such as the percutaneous CT-guided radiofrequency (RF) ablation, CT - guided core-drill excision, arthroscopic removal etc. are gaining popularity. Such methods are less morbid because only precision instruments are used for tissue dissection and ablation of the tumor. Also, said to reduce the cost, hospital stay and recovery time. However, there is still a controversy regarding their success rates which varies from $70-100 \%{ }^{4}$.

It is well known that recurrence of $\mathrm{OO}$ is likely when excision is incomplete ${ }^{13}$. Especially, recurrence after RF ablation has been reported in up to $24 \%$ of patients and most of them in the first 7 months after treatment ${ }^{12}$. Hence, en bloc surgical excision remains as the standard treatment of choice.

The case of IAOO described here is a longstanding one and clinically presented after hypertrophic structural changes occurred in the proximal femur, mimicking a case of primary hip joint pathology. The hypertrophic changes observed here such as enlargement of the proximal femoral epiphysis along with proximal femur, including femoral neck and $1 / 2$ an inch limb lengthening are not described in literature so far. After total excision of the nidus by en bloc excision patient did not have any recurrence.

\section{Conclusions}

IAOO presents differently than extraarticular $\mathrm{OO}$ and can pose difficulty in diagnosis as well as treatment. This is apparent in the case presented in which pain in the hip, lengthening of the limb along with structural change of hypertrophy of proximal femur was misleading. Surgeons must be aware that in such cases careful approach using right diagnostic tools has to be adopted in order to avoid delays in the diagnosis and 
treatment. Finally, surgical excision (en bloc resection) with on table confirmation by imaging and later by histological studies perhaps is the treatment of choice as minimally invasive techniques have yet to prove their efficacy equivalent to surgical excision. Recurrence is not seen after total excision/eradication of the nidus.

\section{Abbreviations}

CT: computed tomography; NSAIDs: Non-steroidalanti-in flammatory drugs; OO: osteoidosteoma; IAOO: Intraarticular Osteoid Osteoma;RF: radiofrequency.

\section{Consent}

Written informed consent was obtained from the patient for publication of this case report and accompanying images. A copy of the written consent is available for review

\section{References}

[1]. Bergstrand H: Über eine eigenartige, wahrscheinlich bisher nicht beschreibene osteoblastische Krankheit in den langen Knochen der Hand und des Fusses. Acta Radiol 1930, 11:596-613.

[2]. Cassar-Pullicino VN, McCall IW, Wan S: Intra-articular osteoidosteoma. Clin Radiol 1992, 45:153-160.

[3]. Regan MW, Galey JP, Oakeshott RD: Recurrent osteoid osteoma. Case report with a ten-year asymptomatic interval. Clin Orthop Relat Res 1990, 253:221-224.

[4]. Papathanassiou ZG, Megas P, Petsas T, Papachristou DJ, Nilas J, Siablis D: Osteoid osteoma: diagnosis and treatment. Orthopedics 2008, 31:1118.

[5]. Edeiken J,DePalma AF,Hodes PJ. Osteoid osteoma (roentgenographic emphasis). Clin Orthop 1966;49:201-206.

[6]. Kattapuram SV,Kushner DC,Phillips WC,Rosenthal DI. Osteoid osteoma:an unusual cause of articular pain. Radiology 1983;147:383-387.

[7]. Resnick D, Kyriakos M, Greenway G: Diagnosis of bone and joints disorders. Volume 4. 2nd edition. Edited by Resnick DNiwayama G. Saunders; 1988::3616- 3888.

[8]. Alan ES,Ramiro JH:Intracapsular Osteoid osteoma of the proximal femur:Findings on plain film and CT.American Journal Of Roentgenology 1990.154;1241-1244.

[9]. Szendroi M, Kollo K, Antal I, Lakatos J, Szoke G: Intraarticular osteoid osteoma: clinical features, imaging results, and comparison with extraarticular localization. J Rheumatol 2004, 31:957-964.

[10]. Kneisl JS, Simon MA: Medical management compared with operative treatment for osteoid-osteoma. J Bone Joint Surg Am 1992, 74:179-185.

[11]. Yildiz Y, Bayrakci K, Altay M, Saglik Y: Osteoid osteoma: the results of surgical treatment.Int Orthop 2001, 25:119-122.

[12]. Cantwell CP, Obyrne J, Eustace S: Current trends in treatment of osteoid osteoma with an emphasis on radiofrequency ablation. Eur Radiol 2004, 14:607-617.

[13]. Norman A: Persistence or recurrence of pain: a sign of surgical failure is osteoid-osteoma. Clin Orthop Relat Res 1978, 130:263-266.

\section{Legends for $\square$ gures}

Figure: 1,2 Plain radiograph Anteroposterior and Lateral view respectively showing an oval nidus surrounded by a radiolucent ring at the femoral neck right side along with hypertrophy of the proximal femur.

Figure: 3, 4 CT and MRI representation of the lesion respectively.

Figure: 5 Anterolateral exposure of right proximal femur showing flakes of synovial fluid and inflammed tissue. TFL-Tensor fascia lata, GM-Gluteus medius, FSF-Flakes of synovial fluid (thickened), JC-Joint capsule(opened).

Figure: 6 ' $\mathrm{C}$ ' arm Image showing localization of the lesion using ' $\mathrm{K}$ ' wire.

Figure 7 Showing excised nidus along with block of normal bone.

Figure: 8 \& 9 Showing $c$ arm images of anteroposterior and lateral views respectively for on table confirmation of complete excision of the tumor.

Figure: 10 Histopathologic features of osteoid osteoma. Nidus displaying well mineralized trabeculae of woven bone lined by numerous active osteoblasts and multinucleated giant cell-like osteoclasts. Hematoxylin and eosin (X100).

Figure: 11 Showing $x$ ray after one year with no evidence of recurrence. Bone is well moulded.

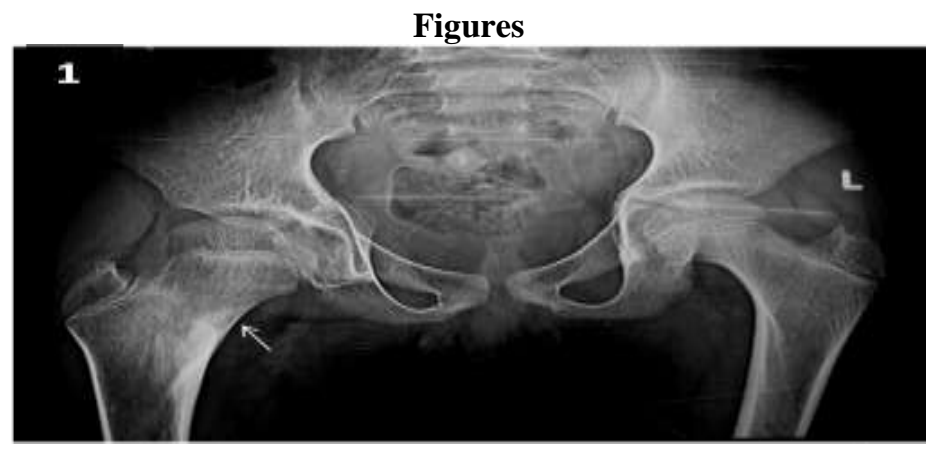



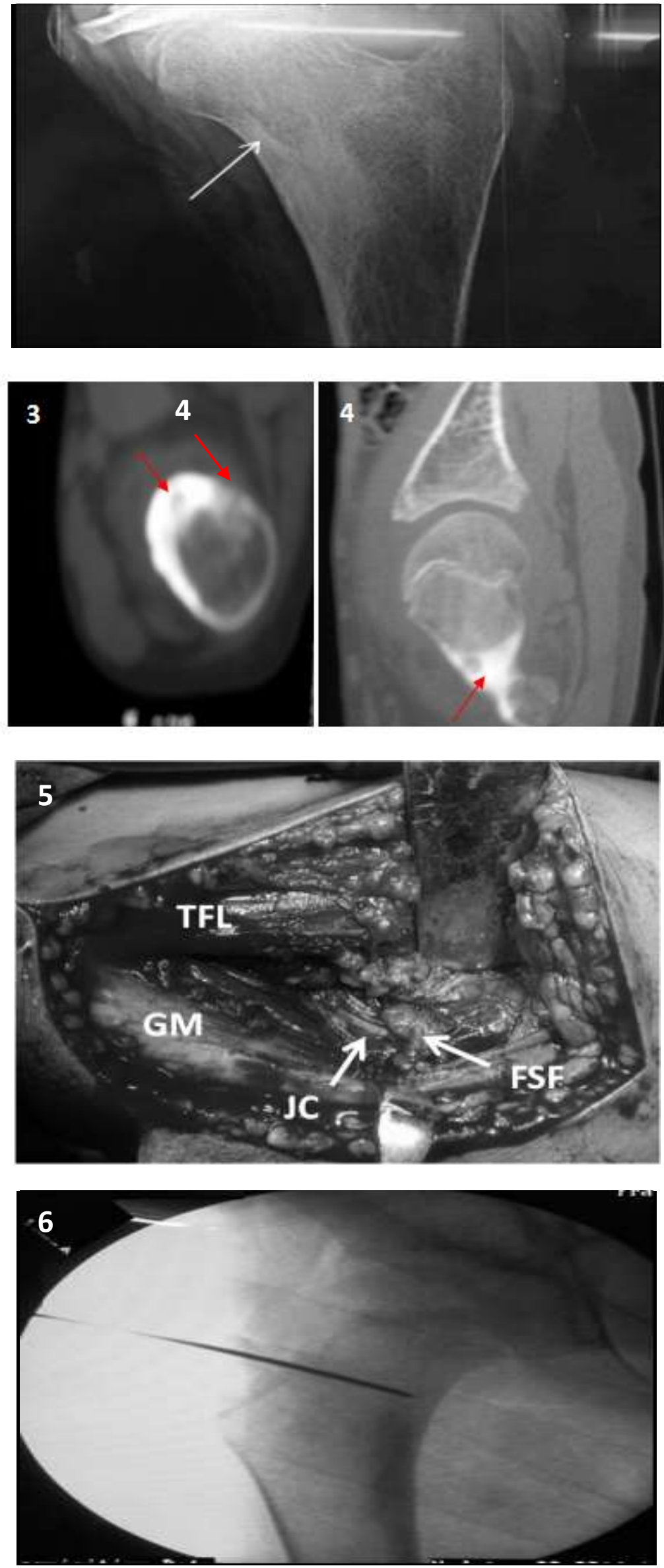

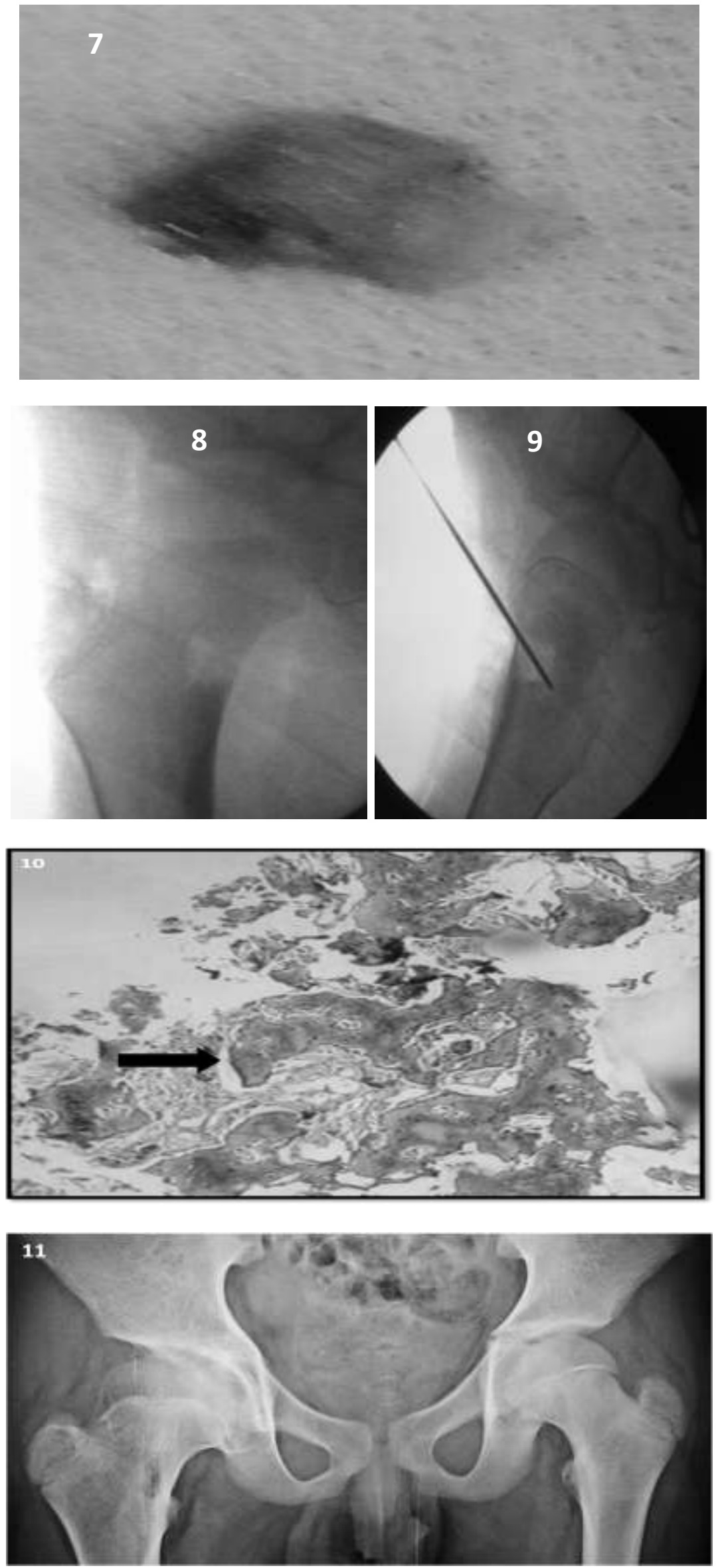